\title{
Moments Match between the KPZ Equation and the Airy Point Process ${ }^{\star}$
}

\author{
Alexei BORODIN ${ }^{\dagger}$ and Vadim GORIN ${ }^{\dagger \ddagger}$
}

$\dagger$ Department of Mathematics, Massachusetts Institute of Technology, USA

E-mail: borodin@math.mit.edu,vadicgor@gmail.com

$\ddagger$ Institute for Information Transmission Problems of Russian Academy of Sciences, Russia

Received August 09, 2016, in final form October 21, 2016; Published online October 26, 2016

http://dx.doi.org/10.3842/SIGMA.2016.102

\begin{abstract}
The results of Amir-Corwin-Quastel, Calabrese-Le Doussal-Rosso, Dotsenko, and Sasamoto-Spohn imply that the one-point distribution of the solution of the KPZ equation with the narrow wedge initial condition coincides with that for a multiplicative statistics of the Airy determinantal random point process. Taking Taylor coefficients of the two sides yields moment identities. We provide a simple direct proof of those via a combinatorial match of their multivariate integral representations.
\end{abstract}

Key words: KPZ equation; Airy point process

2010 Mathematics Subject Classification: 60B20; 60H15; 33C10

\section{Introduction}

Since Tracy and Widom's discovery of the ASEP solvability eight years ago [18, 19, 20], the relationship between the "determinantal" and "non-determinantal" solvable models in the $(1+1)$ dimensional KPZ (Kardar-Parisi-Zhang) universality class has largely remained a mystery. One step towards solving this mystery is the celebrated result of Amir-Corwin-Quastel [1], Calabrese-Le Doussal-Rosso [8], Dotsenko [10], and Sasamoto-Spohn [17], that provides an explicit expression for the distribution (or its Laplace transform) of one-point value of the solution of the KPZ equation with the so-called narrow wedge initial condition. It can be re-interpreted as saying that this Laplace transform coincides with the average of a multiplicative statistics of the Airy determinantal random point process. Although this restatement seems to be known to experts, we couldn't find it in this form in the literature, so we give an exact formulation as Theorem 2.1 below. Such a result is very useful as it immediately implies that this solution of the KPZ equation asymptotically at large times has the GUE Tracy-Widom distribution, which is a display of the KPZ universality, cf. Corwin's survey [9].

Finding other facts of similar nature has been a challenge so far.

Imamura and Sasamoto [12] proved a similar statement for the O'Connell-Yor semi-discrete Brownian directed polymer. Unfortunately, the associated determinantal point process was not governed by a positive measure. Still, taking the edge limit of this process, they were able to recover Theorem 2.1. Another representation of the Laplace transform of the O'Connell-Yor partition function as the average of a multiplicative functional over a signed determinantal point process can be found in [14].

Very recently, one of the authors found in [4] an identity that relates a single point height distribution of the (higher spin inhomogeneous) stochastic six vertex model in a quadrant on

*This paper is a contribution to the Special Issue on Asymptotics and Universality in Random Matrices, Random Growth Processes, Integrable Systems and Statistical Physics in honor of Percy Deift and Craig Tracy. The full collection is available at http://www.emis.de/journals/SIGMA/Deift-Tracy.html 
one side, and multiplicative statistics of the Macdonald measures on the other. The ASEP limit of this identity was worked out in [7]. Taking the KPZ limit of both leads to Theorem 2.1 again.

The goal of this note is to look at Theorem 2.1 from the point of view of moments, rather than the corresponding distributions. One can study both the KPZ equation and the Airy point process via their exponential moments. Those are computationally tractable but they are of limited mathematical use because the corresponding moment problems are indeterminate. Still, on the KPZ side physicists were able to consistently use the moments to access the distributions via the (non-rigorous) replica trick, see [10] and [8] for early examples.

Our Theorem 2.2 proves the moments identity that corresponds to Theorem 2.1. The argument is a combinatorial match between known multivariate integral representations of the moments on both sides. Interestingly, these integral representations were known long before $[1,8,10,17]$, but their similarity had not been exploited. We are hoping that the moments point of view will be beneficial for finding other similar correspondence.

We did attempt to extend the moments correspondence to a two-point identity, as integral representations on both side are again known. Unfortunately, we have not been successful in that so far.

\section{The one-point equality}

Let $\mathfrak{a}_{1} \geq \mathfrak{a}_{2} \geq \mathfrak{a}_{3} \geq \cdots$ be points of the Airy point process ${ }^{1}$ at $\beta=2$ (see, e.g., $[2,11]$ ) which is a determinantal point process on $\mathbb{R}$ with correlation kernel

$$
K_{\text {Airy }}(x, y)=\frac{\operatorname{Ai}(x) \operatorname{Ai}^{\prime}(y)-\operatorname{Ai}^{\prime}(x) \operatorname{Ai}(y)}{x-y}=\int_{0}^{\infty} \operatorname{Ai}(x+a) \operatorname{Ai}(y+a) d a .
$$

Here $\operatorname{Ai}(x)$ is the Airy function.

From the opposite direction, let $\mathcal{Z}(T, X)$ denote the solution of the stochastic heat equation (see, e.g., $[9,16])$

$$
\frac{\partial}{\partial T} \mathcal{Z}=\frac{1}{2} \frac{\partial^{2}}{\partial X^{2}} \mathcal{Z}-\mathcal{Z} \dot{\mathcal{W}}, \quad \mathcal{Z}(0, X)=\delta(X=0)
$$

where $\dot{\mathcal{W}}$ is the space-time white noise. $H:=-\log (\mathcal{Z})$ is the Hopf-Cole solution of the KardarParisi-Zhang stochastic partial differential equation with the narrow-edge initial data.

The following statement is a reformulation of results of $[1,8,10,17]$.

Theorem 2.1. Set $\frac{T}{2}=C^{3}$. Then for each real $C>0, u \geq 0$ we have

$$
\mathbb{E}_{\text {Airy }}\left[\prod_{k=1}^{\infty} \frac{1}{1+u \exp \left(C \mathfrak{a}_{k}\right)}\right]=\mathbb{E}_{\mathrm{KPZ}}\left[\exp \left(-u \mathcal{Z}(T, 0) \exp \left(\frac{T}{24}\right)\right)\right] .
$$

On the other hand, we could not find the following statement in the existing literature.

Theorem 2.2. Set $\frac{T}{2}=C^{3}$, and let $h_{k}\left(x_{1}, x_{2}, \ldots\right)=\sum_{i_{1} \leq i_{2} \leq \cdots \leq i_{k}} x_{i_{1}} x_{i_{2}} \cdots x_{i_{k}}$ be the complete symmetric homogeneous function in variables $x_{1}, x_{2}, \ldots$ Then for each $C>0, k=1,2, \ldots$ we have

$$
\mathbb{E}_{\text {Airy }}\left[h_{k}\left(\exp \left(C \mathfrak{a}_{1}\right), \exp \left(C \mathfrak{a}_{2}\right), \ldots\right)\right]=\mathbb{E}_{\mathrm{KPZ}}\left[\frac{\mathcal{Z}(T, 0)^{k}}{k !}\right] \exp \left(k \frac{T}{24}\right) .
$$

\footnotetext{
${ }^{1}$ It should not be confused with Airy 2 process; the latter is a random continuous curve rather than a random point process.
} 
Our proof of Theorem 2.2 is based on direct comparison of contour integral formulas: for the right-hand side of (2) such formula is known as a solution for the attractive delta Bose gas equation, cf. the discussion in [6, Section 6.2], while for the left-hand side it can be computed through the Laplace transform of the correlation kernel $K_{\text {Airy }}$.

Remark 2.3. Expanding formally the result of Theorem 2.1 into power series in $u$ and evaluating the coefficients, one gets the result of Theorem 2.2 and vice versa. However, these theorems are not equivalent: The $u$ power series expansion of the left-hand side of (2.1) fails to converge for any $u \neq 0$. Below we provide two different proofs for Theorems 2.1 and 2.2 , respectively.

The following corollary is present in $[1,8,10,17]$, but it seems reasonable for us to give a proof using Theorem 2.1 above only, without appealing to the explicit evaluation of either side.

Corollary 2.4. The following convergence in distribution holds:

$$
\lim _{T \rightarrow+\infty}\left[\left(\frac{2}{T}\right)^{1 / 3}\left(\ln (Z(T, 0))+\frac{T}{24}\right)\right]=\mathfrak{a}_{1} .
$$

Proof. Take $a \in \mathbb{R}$ and set $u=\exp \left(-(T / 2)^{1 / 3} a\right)$. Then the left-hand side of (1) is

$$
\mathbb{E}\left[\prod_{k=1}^{\infty} \frac{1}{1+\exp \left((T / 2)^{1 / 3}\left(\mathfrak{a}_{k}-a\right)\right)}\right]
$$

We claim that the random variable under expectation in (3) almost surely converges as $T \rightarrow \infty$ to the indicator function of the event $\mathfrak{a}_{1}<a$. Indeed, if $\mathfrak{a}_{1}>a$, then the expression in (3) converges to 0 as $T \rightarrow \infty$. On the other hand, if $\mathfrak{a}_{1}<a$, then (taking into the account that $\sum_{k=1}^{\infty} \exp \left(\mathfrak{a}_{k}\right)$ is almost surely finite, as follows from the finiteness of its expectation, which is explicitly known, see, e.g., [15, Section 2.6.1]) the same expression converges to 1 . Since the random variable under expectation is almost surely between 0 and 1 , the almost sure convergence implies convergence of expectations, and therefore

$$
\lim _{T \rightarrow+\infty} \mathbb{E}\left[\prod_{k=1}^{\infty} \frac{1}{1+\exp \left((T / 2)^{1 / 3}\left(\mathfrak{a}_{k}-a\right)\right)}\right]=\operatorname{Prob}\left(\mathfrak{a}_{1}<a\right) .
$$

On the other hand, the right side of (1) is

$$
\mathbb{E}\left[\exp \left(-\exp \left((T / 2)^{1 / 3}\left(\frac{\ln (\mathcal{Z}(T, 0))+T / 24}{(T / 2)^{1 / 3}}-a\right)\right)\right)\right]
$$

Observe that for any random variable $\xi$, the expression $\exp \left(-\exp \left((T / 2)^{1 / 3}(\xi-a)\right)\right)$ is almost surely between 0 and 1 , converges to 0 as $T \rightarrow+\infty$ if $\xi>a$, and converges to 1 if $\xi<a$. Therefore, using the fact that $\mathfrak{a}_{1}$ has a continuous distribution, (5) as $T \rightarrow \infty$ behaves as (see, e.g., [6, Lemma 4.1.39] for more details)

$$
\operatorname{Prob}\left(\frac{\ln (\mathcal{Z}(T, 0))+T / 24}{(T / 2)^{1 / 3}}<a\right)+o(1)
$$

Equating (4) to (6) we are done. 


\section{Proofs of Theorems 2.1 and 2.2}

Proof of Theorem 2.1. The expectation $\mathbb{E} \exp (-u \mathcal{Z}(T, 0)$ admits a formula as a Fredholhm determinant, which was discovered in [1, 8, 10, 17]. Following [5, Section 2.2.1], this formula reads

$$
\mathbb{E}[\exp (-u \mathcal{Z}(T, 0) \exp (T / 24))]=1+\sum_{L=1}^{\infty} \frac{(-1)^{L}}{L !} \int_{0}^{\infty} d x_{1} \cdots \int_{0}^{\infty} d x_{L} \operatorname{det}\left[K_{u}\left(x_{i}, x_{j}\right)\right]_{i, j=1}^{L},
$$

where

$$
K_{u}\left(x, x^{\prime}\right)=\int_{-\infty}^{\infty} \frac{d r}{1+\frac{1}{u} \exp \left((T / 2)^{1 / 3} r\right)} \operatorname{Ai}(x-r) \operatorname{Ai}\left(x^{\prime}-r\right) .
$$

On the other hand, the left-hand side of (1) is a multiplicative function of a determinantal point process and, therefore, also admits a Fredholm determinant formula, see, e.g., [3, equation (2.4)]:

$$
\begin{aligned}
& \mathbb{E}\left[\prod_{k=1}^{\infty} \frac{1}{1+u \exp \left(C \mathfrak{a}_{k}\right)}\right]=\operatorname{det}\left[1-\left(1-\frac{1}{1+u \exp (C r)}\right) K_{\text {Airy }}\left(r, r^{\prime}\right)\right]_{L_{2}(\mathbb{R})} \\
& =1+\sum_{L=1}^{\infty} \frac{(-1)^{L}}{L !} \int_{-\infty}^{\infty} d y_{1} \cdots \int_{-\infty}^{\infty} d y_{L}\left(\prod_{k=1}^{L} \frac{1}{1+\frac{1}{u} \exp \left(-C y_{k}\right)}\right) \operatorname{det}\left[K_{\text {Airy }}\left(y_{i}, y_{j}\right)\right]_{i, j=1}^{L} .
\end{aligned}
$$

One immediately sees that upon the change of variables $r_{i}=-y_{i}$ and identification $\frac{T}{2}=C^{3}$, the formulas (7) and (8) are the same.

Proof of Theorem 2.2. The moments $\mathbb{E} \mathcal{Z}(T, 0)^{k}$ are known through solving the attractive delta Bose gas equation, cf. the discussion in [6, Section 6.2]. Following [5, Lemma 4.1], we have

$$
\mathbb{E}\left[\mathcal{Z}(T, 0)^{k}\right]=\int_{a_{1}-\mathrm{i} \infty}^{a_{1}+\mathrm{i} \infty} \frac{d z_{1}}{2 \pi \mathrm{i}} \cdots \int_{a_{k}-\mathrm{i} \infty}^{a_{k}+\mathrm{i} \infty} \frac{d z_{k}}{2 \pi \mathrm{i}} \prod_{1 \leq A<B \leq k} \frac{z_{A}-z_{B}}{z_{A}-z_{B}-1} \cdot \prod_{j=1}^{k} \exp \left(\frac{T}{2} z_{j}^{2}\right),
$$

where the real numbers $a_{1}, \ldots, a_{k}$ satisfy $a_{1} \gg a_{2} \gg \cdots \gg a_{k}$. It is convenient for us to modify the contours of integration in (9) to the imaginary axis $i \mathbb{R}$. One collects certain residues in such a deformation, and the final result is read from [5, equation (13)] to be

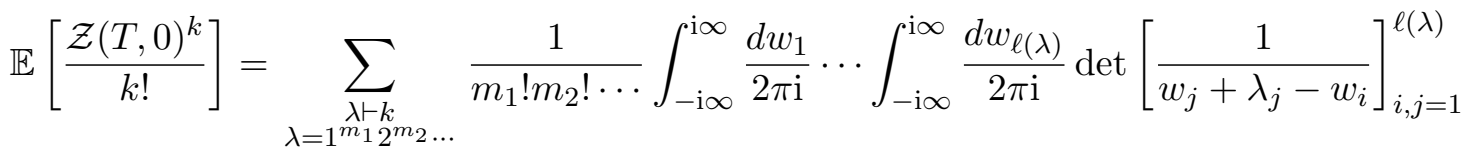

$$
\begin{aligned}
& \times \prod_{j=1}^{\ell(\lambda)} \exp \left(\frac{T}{2}\left(w_{j}^{2}+\left(w_{j}+1\right)^{2}+\cdots+\left(w_{j}+\lambda_{j}-1\right)^{2}\right)\right),
\end{aligned}
$$

where $\lambda=\left(\lambda_{1} \geq \lambda_{2} \geq \ldots\right)$ is a partition of $k$ and $\ell(\lambda)$ is the number of non-zero parts $\lambda_{j}$.

Let us now produce a similar expression for the left-hand side of (2). Define the Laplace transform of the correlation functions of the Airy point process through

$$
R\left(c_{1}, \ldots, c_{n}\right)=\int_{\mathbb{R}^{n}} e^{(c \cdot x)} \operatorname{det}\left[K_{\text {Airy }}\left(x_{i}, x_{j}\right)\right]_{i, j=1}^{n} d x_{1} \cdots d x_{n}, \quad c_{1}, c_{2}, \ldots, c_{n}>0 .
$$

The definition of the Airy point process implies that for a partition $\lambda=\left(\lambda_{1} \geq \lambda_{2} \cdots \geq \lambda_{\ell}\right)=$ $1^{m_{1}} 2^{m_{2}} \cdots$ one has

$$
\mathbb{E}\left[m_{\lambda}\left(\exp \left(C \mathfrak{a}_{1}\right), \exp \left(C \mathfrak{a}_{2}\right), \ldots\right)\right]=\frac{1}{m_{1} ! m_{2} ! \cdots} R\left(C \lambda_{1}, \ldots, C \lambda_{\ell}\right),
$$


where $m_{\lambda}\left(y_{1}, y_{2}, \ldots\right)$ is the monomial symmetric function in variables $y_{1}, y_{2}, \ldots$, as in [13, Chapter I]. Expanding $h_{k}$ into linear combination of $m_{\lambda}$ 's, we can then write

$$
\mathbb{E}\left[h_{k}\left(\exp \left(C \mathfrak{a}_{1}\right), \exp \left(C \mathfrak{a}_{2}\right), \ldots\right)\right]=\sum_{\substack{\lambda \vdash k \\ \lambda=1^{m_{1}} 2^{m_{2} \ldots}}} \frac{1}{m_{1} ! m_{2} ! \cdots} R\left(C \lambda_{1}, \ldots, C \lambda_{\ell(\lambda)}\right),
$$

where the summation goes over all partitions of $k$. Comparing (11) with (10), we see that it remains to identify the contour integrals over imaginary axis in (10) with $R\left(C \lambda_{1}, \ldots, C \lambda_{\ell(\lambda)}\right)$.

The rest is based on the following identity that can be found in [15, Lemma 2.6]:

$$
\int_{-\infty}^{+\infty} e^{x z} \operatorname{Ai}(z+a) \operatorname{Ai}(z+b) d z=\frac{1}{2 \sqrt{\pi x}} \exp \left(\frac{x^{3}}{12}-\frac{a+b}{2} x-\frac{(a-b)^{2}}{4 x}\right), \quad x>0 .
$$

Its immediate corollary is (we use an agreement $z_{n+1}=z_{1}$ and $s_{n+1}=s_{1}$ here, and also assume $\left.c_{1}, \ldots, c_{n}>0\right)$

$$
\begin{aligned}
\mathcal{E}\left(c_{1}, \ldots, c_{n}\right):= & \int_{\mathbb{R}^{n}} e^{(c \cdot z)} \prod_{i=1}^{n} K_{\text {Airy }}\left(z_{i}, z_{i+1}\right) d z=\frac{1}{2^{n} \pi^{n / 2}} \frac{e^{\sum c_{i}^{3} / 12}}{\prod_{i=1}^{n} \sqrt{c_{i}}} \\
& \times \int_{s_{1} \geq 0} \cdots \int_{s_{n} \geq 0} \exp \left(-\sum_{i=1}^{n} \frac{\left(s_{i}-s_{i+1}\right)^{2}}{4 c_{i}}-\sum_{i=1}^{n} \frac{s_{i}+s_{i+1}}{2} c_{i}\right) \prod_{i=1}^{n} d s_{i} .
\end{aligned}
$$

Using the Gaussian integrals in variables $z_{1}, \ldots, z_{n}$, the last formula is converted into

$$
\begin{aligned}
\mathcal{E}\left(c_{1}, \ldots, c_{n}\right)= & \frac{1}{(2 \pi)^{n}} e^{\sum c_{i}^{3} / 12} \int_{s_{1} \geq 0} d s_{1} \cdots \int_{s_{n} \geq 0} d s_{n} \int_{z_{1} \in \mathbb{R}} d z_{1} \cdots \int_{z_{n} \in \mathbb{R}} d z_{n} \\
& \times \exp \left(\sum_{i=1}^{n}\left(-c_{i} z_{i}^{2}+\mathrm{i}\left(z_{i}-z_{i+1}\right) s_{i}-\left(c_{i}+c_{i+1}\right) s_{i} / 2\right)\right) .
\end{aligned}
$$

Since i $\left(z_{i}-z_{i+1}\right)$ has zero real part, we can integrate over $s_{i}$ in (12), arriving at the formula:

$$
\mathcal{E}\left(c_{1}, \ldots, c_{n}\right)=\frac{e^{\sum x_{i}^{3} / 12}}{(2 \pi)^{n}} \int_{z_{1} \in \mathbb{R}} \cdots \int_{z_{n} \in \mathbb{R}} \exp \left(-\sum_{i=1}^{n} c_{i} z_{i}^{2}\right) \prod_{i=1}^{n} \frac{d z_{i}}{-\mathrm{i}\left(z_{i}-z_{i+1}\right)+\frac{c_{i}+c_{i+1}}{2}} .
$$

We can now write the formula for $R\left(c_{1}, \ldots, c_{n}\right)$ (we subdivide a permutation into cycles, use (13) and then combine back):

$$
\begin{aligned}
& R\left(c_{1}, \ldots, c_{n}\right)=\int_{\mathbb{R}^{n}} d x_{1} \cdots d x_{n} \sum_{\sigma \in \mathfrak{S}(n)}(-1)^{\sigma} \prod_{j=1}^{n} e^{x_{j} c_{j}} K_{\text {Airy }}\left(x_{j}, x_{\sigma(j)}\right) \\
& =\frac{e^{\sum c_{i}^{3} / 12}}{(2 \pi)^{n}} \int_{z_{1} \in \mathbb{R}} \cdots \int_{z_{n} \in \mathbb{R}} \exp \left(-\sum_{i=1}^{n} c_{i} z_{i}^{2}\right) \sum_{\sigma \in \mathfrak{S}(n)}(-1)^{\sigma} \prod_{i=1}^{n} \frac{d z_{i}}{-\mathrm{i}\left(z_{i}-z_{\sigma(i)}\right)+\frac{c_{i}+c_{\sigma(i)}}{2}} \\
& =\frac{e^{\sum c_{i}^{3} / 12}}{(2 \pi)^{n}} \int_{z_{1} \in \mathbb{R}} d z_{1} \cdots \int_{z_{n} \in \mathbb{R}} d z_{n} \exp \left(-\sum_{i=1}^{n} c_{i} z_{i}^{2}\right) \operatorname{det}\left[\frac{1}{\left(-\mathrm{i} z_{i}+\frac{c_{i}}{2}\right)+\left(\mathrm{i} z_{j}+\frac{c_{j}}{2}\right)}\right]_{i, j=1}^{n} .
\end{aligned}
$$

Remark 3.1. We can use the Cauchy determinant formula

$$
\operatorname{det}\left[\frac{1}{a_{i}+b_{j}}\right]_{i, j=1}^{n}=\prod_{i=1}^{n} \frac{1}{a_{i}+b_{i}} \prod_{1 \leq i<j \leq n} \frac{\left(a_{i}-a_{j}\right)\left(b_{i}-b_{j}\right)}{\left(a_{i}+b_{j}\right)\left(a_{j}+b_{i}\right)}
$$

with $a_{i}=-\mathrm{i} z_{i}+c_{i} / 2, b_{i}=\mathrm{i} z_{i}+c_{i} / 2$ to simplify the last determinant. 
We now take a partition $\lambda \vdash k$ with $\ell(\lambda)=n$, set $c_{i}=C \lambda_{i}$ and make a change of variables

$$
\mathrm{i} z_{j}=C w_{j}+\frac{C \lambda_{j}}{2}-\frac{C}{2}
$$

to get (note that we deformed the contours to the imaginary axis; we do not pick up any residues in such a deformation)

$$
\begin{aligned}
R\left(C \lambda_{1}, \ldots, C \lambda_{n}\right)= & \frac{\exp \left(C^{3} \sum_{i=1}^{n} \lambda_{i}^{3} / 12\right)}{(2 \pi \mathrm{i})^{n}} \int_{-\mathrm{i} \infty}^{\mathrm{i} \infty} d w_{1} \cdots \int_{-\mathrm{i} \infty}^{\mathrm{i} \infty} d w_{n} \\
& \times \exp \left(C^{3} \sum_{i=1}^{n} \lambda_{i}\left(w_{i}+\lambda_{i} / 2-1 / 2\right)^{2}\right) \operatorname{det}\left[\frac{1}{w_{j}+\lambda_{j}-w_{i}}\right]_{i, j=1}^{n} .
\end{aligned}
$$

It remains to simplify the exponents:

$$
\begin{aligned}
\exp & {\left[C^{3} \sum_{i=1}^{n}\left(\frac{\lambda_{i}^{3}}{12}+\lambda_{i}\left(w_{i}+\frac{\lambda_{i}}{2}-\frac{1}{2}\right)^{2}\right)\right] } \\
& =\exp \left[C^{3} \sum_{i=1}^{n}\left(\lambda_{i} w_{i}^{2}+\frac{\lambda_{i}^{3}}{3}+\frac{\lambda_{i}}{4}+w_{i} \lambda_{i}^{2}-w_{i} \lambda_{i}-\frac{\lambda_{i}^{2}}{2}\right)\right] \\
& =\exp \left[C^{3} \sum_{i=1}^{n}\left(\lambda_{i} w_{i}^{2}+\lambda_{i}\left(\lambda_{i}-1\right) w_{i}+\frac{\lambda_{i}\left(\lambda_{i}-1\right)\left(2 \lambda_{i}-1\right)}{6}-\frac{\lambda_{i}}{12}\right)\right] \\
& =\exp \left[C^{3} \sum_{i=1}^{n}\left(w_{i}^{2}+\left(w_{i}+1\right)^{2}+\cdots+\left(w_{i}+\lambda_{i}-1\right)^{2}\right)-C^{3} \frac{k}{12}\right] .
\end{aligned}
$$

Combining (11) with (14), (15) and identifying $C^{3}=\frac{T}{2}$. we arrive at (10) multiplied by $\exp (-k T / 24)$.

\section{Acknowledgements}

A.B. was partially supported by the NSF grants DMS-1056390 and DMS-1607901. V.G. was partially supported by the NSF grant DMS-1407562 and by the Sloan Research Fellowship.

\section{References}

[1] Amir G., Corwin I., Quastel J., Probability distribution of the free energy of the continuum directed random polymer in $1+1$ dimensions, Comm. Pure Appl. Math. 64 (2011), 466-537, arXiv:1003.0443.

[2] Anderson G.W., Guionnet A., Zeitouni O., An introduction to random matrices, Cambridge Studies in Advanced Mathematics, Vol. 118, Cambridge University Press, Cambridge, 2010.

[3] Borodin A., Determinantal point processes, in The Oxford Handbook of Random Matrix Theory, Oxford University Press, Oxford, 2011, 231-249, arXiv:0911.1153.

[4] Borodin A., Stochastic higher spin six vertex model and Macdonald measures, arXiv:1608.01553.

[5] Borodin A., Bufetov A., Corwin I., Directed random polymers via nested contour integrals, Ann. Physics 368 (2016), 191-247, arXiv:1511.07324.

[6] Borodin A., Corwin I., Macdonald processes, Probab. Theory Related Fields 158 (2014), 225-400, arXiv:1111.4408.

[7] Borodin A., Olshanski G., The ASEP and determinantal point processes, arXiv:1608.01564.

[8] Calabrese P., Le Doussal P., Rosso A., Free-energy distribution of the directed polymer at high temperature, Europhys. Lett. 90 (2010), 20002, 6 pages, arXiv:1002.4560. 
[9] Corwin I., The Kardar-Parisi-Zhang equation and universality class, Random Matrices Theory Appl. 1 (2012), 1130001, 76 pages, arXiv:1106.1596.

[10] Dotsenko V., Bethe ansatz derivation of the Tracy-Widom distribution for one-dimensional directed polymers, Europhys. Lett. 90 (2010), 20003, 5 pages, arXiv:1003.4899.

[11] Forrester P.J., Log-gases and random matrices, London Mathematical Society Monographs Series, Vol. 34, Princeton University Press, Princeton, NJ, 2010.

[12] Imamura T., Sasamoto T., Determinantal structures in the O'Connell-Yor directed random polymer model, J. Stat. Phys. 163 (2016), 675-713, arXiv:1506.05548.

[13] Macdonald I.G., Symmetric functions and Hall polynomials, 2nd ed., Oxford Mathematical Monographs, The Clarendon Press, Oxford University Press, New York, 1995.

[14] O'Connell N., Directed polymers and the quantum Toda lattice, Ann. Probab. 40 (2012), 437-458, arXiv:0910.0069.

[15] Okounkov A., Generating functions for intersection numbers on moduli spaces of curves, Int. Math. Res. Not. 2002 (2002), 933-957, math.AG/0101201.

[16] Quastel J., Introduction to KPZ, in Current Developments in Mathematics, 2011, Int. Press, Somerville, MA, 2012, 125-194, available at http://math.arizona.edu/ mathphys/school_2012/IntroKPZ-Arizona.pdf.

[17] Sasamoto T., Spohn H., One-dimensional Kardar-Parisi-Zhang equation: an exact solution and its universality, Phys. Rev. Lett. 104 (2010), 230602, 4 pages, arXiv:1002.1883.

[18] Tracy C.A., Widom H., A Fredholm determinant representation in ASEP, J. Stat. Phys. 132 (2008), 291300, arXiv:0804.1379.

[19] Tracy C.A., Widom H., Integral formulas for the asymmetric simple exclusion process, Comm. Math. Phys. 279 (2008), 815-844, Erratum, Comm. Math. Phys. 304 (2011), 875-878, arXiv:0704.2633.

[20] Tracy C.A., Widom H., Asymptotics in ASEP with step initial condition, Comm. Math. Phys. 290 (2009), 129-154, arXiv:0807.1713. 